\title{
Killian Jamieson Pouch: A Rarer Cervical Diverticulum
}

\author{
Prafulla Sakhare, Binhi Desai, Bhagyashree Bokare, Seema Patel, Apurva Pawde
}

\begin{abstract}
Killian Jamieson diverticulum (KJD) is lesser common than Zenker's diverticulum (ZD). Various treatment modalities have been recommended but traditional surgical treatment has been recommended for a symptomatic KJD.

Recently, traditional open surgery for a symptomatic KJD is being challenged by the development of new endoscopic techniques and devices.

We document a rare report of a left KJD diagnosed on barium esophagogram and flexible endoscopy. Diverticulum excision and an esophagomyotomy was done and following treatment the patient's symptoms resolved and he recovered well.
\end{abstract}

Keywords: Killian Jamieson Diverticulum, Zenker's Diverticulum, Cricopharyngeus, Recurrent laryngeal nerve, Surgical excision, Esophagomyotomy.

How to cite this article: Sakhare P, Desai B, Bokare B, Patel S, Pawde A. Killian Jamieson Pouch: A Rarer Cervical Diverticulum. Int J Phonosurg Laryngol 2013;3(2):65-68.

\section{Source of support: Nil}

Conflict of interest: None declared

\section{INTRODUCTION}

Killian Jamieson diverticulum (KJD) is a rare, unusual and unfamiliar cervical esophageal diverticulum. It is an outpouching from lateral wall of proximal cervical esophagus from Killian's space, which is below the cricopharyngeus muscle and lateral to the longitudinal muscle of esophagus. Because of the unfamiliarity and rarity of this entity, a $\mathrm{KJD}$ is often unrecognized and misdiagnosed as a ZD on an endoscopic finding. Accurate differentiation between a ZD and KJD relies on radiological studies, especially the use of a contrast esophagogram. The opening of ZD is seen radiologically directly above the protruding cricopharyngeal 'bar', with the sac of the diverticulum lying posterior to the cervical esophagus on lateral images and in the midline on frontal images. ${ }^{1}$ In contrast, the opening of a Killian Jamieson diverticulum is located just below the level of the cricopharyngeus, with the sac of the diverticulum lying lateral to the cervical esophagus on lateral images.

Very few reports regarding the treatment of KillianJamieson diverticula have been cited in the literature. Due to rarity of cases, the safety of endoscopic treatment has not been established for KJD.

We report a case of 52 years male, who was diagnosed as left KJD on barium esophagogram and flexible endoscopy, whom we managed with surgery via external approach.

\section{CASE REPORT}

A 52 years male, came to our institute with complaints of gradually progressive difficulty in swallowing solids and liquids since 6 months. The dysphagia was more for solids than liquids. He also complained of having noticed a diffuse swelling over left side of neck, approximately the size of a lemon but it would completely reduce on applying pressure over it (Fig. 1).

The patient does not give any history of oral or nasal regurgitation during day or night. There was no history of chronic cough or voice change or respiratory distress. There was also no history of halitosis or reflux symptoms. There was no history of recent weight loss or habitual weight lifting exercises or any addiction (Figs 2A and B to 4).

On examination, there was appearance of diffuse bulge overlying left middle and lower 1/3rd of sternocleidomastoid after swallowing food. The swelling would completely reduce after applying pressure over it. The swelling would not appear after coughing or after valsalva manoeuvre. Rest of the neck was normal. Indirect laryngoscopic examination was also normal. Rest of the otolaryngology examination was also normal.

We did a contrast esophagram, which showed a large contrast filling diverticulum seen to be arising laterally below the cricopharyngeus muscle bar extending further laterally. It had a narrow mouth. Rest esophagus was normal. Following this we did a computed tomography (CT) of neck to do the extent and relations. It showed a $4.5 \times 3.8 \times 1.2 \mathrm{~cm}$ contrast with air fluid filled outpouching on the anterolateral aspect of cervical esophagus. It is related left laterally to sternocleidomastoid and internal jugular vein. It is related posterolaterally to carotid vessels. Inferiorly it has a blind ending at the level of subclavian vein. We also did a flexible esophagoscopy which showed a diverticulum seen to be arising from the anterolateral aspect of cervical esophagus on left side below cricopharyngeal bar. The diverticulum contained some food debris and saliva which were suctioned out. The mucosa was healthy. There was no ulcer or growth.

The provisional diagnosis of a left cervical esophageal diverticulum? Killian Jamieson was made. We posted the patient for left transcervical diverticulum excision with esophageal myotomy. The diverticulum was approached through an oblique incision along the anterior border of the left sternocleidomastoid muscle with the patient's head extended and slightly turned to the right. After dividing the omohyoid muscle, the diverticulum was visualized. It was not adherent to surrounding structures. The diverticulum was dissected up to its base. Left recurrent nerve was identified and seen to be in entering the larynx near the base and carefully preserved. Cricopharyngomyotomy was 


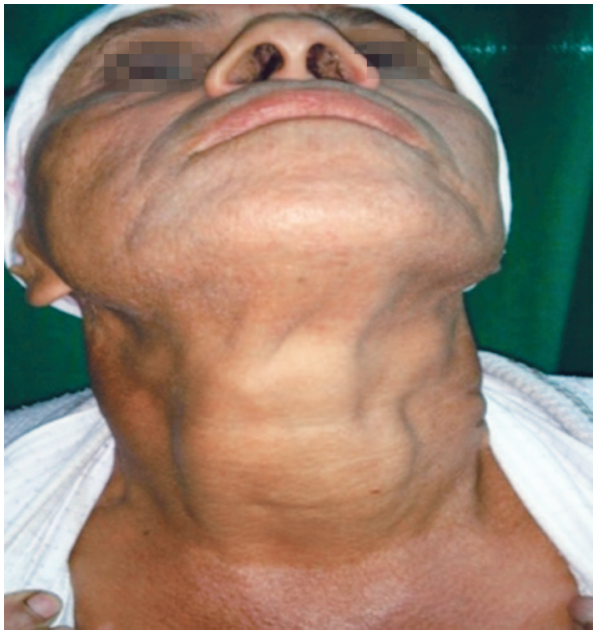

Fig. 1: Diffuse bulge over left middle and lower $1 / 3$ rd of sternocleidomastoid

then performed. Esophagus mucosa was closed with purse string sutures. Neck wound was closed in layers and after placing a closed suction drain. The patient was on Ryle's tube feeding for a period of 14 days. Patient was started clear fluid and advanced to soft diet. The drain was removed on postoperative day 2. Follow-up after 1 month revealed that the patient had no symptoms of dysphagia or neck swelling. There was also no change in voice. On esophagoscopy, there was no diverticulum. The mucosa had completely healed. The patient is under follow-up and is symptom free.

\section{DISCUSSION}

An outpouching from the lateral wall of the proximal cervical esophagus is defined as a Killian-Jamieson diverticulum. These diverticula protrude through a muscular gap in the anterolateral wall of the proximal esophagus, inferior to the cricopharyngeus muscle and superior-lateral to the longitudinal muscle of the esophagus. ${ }^{2}$ This gap was first

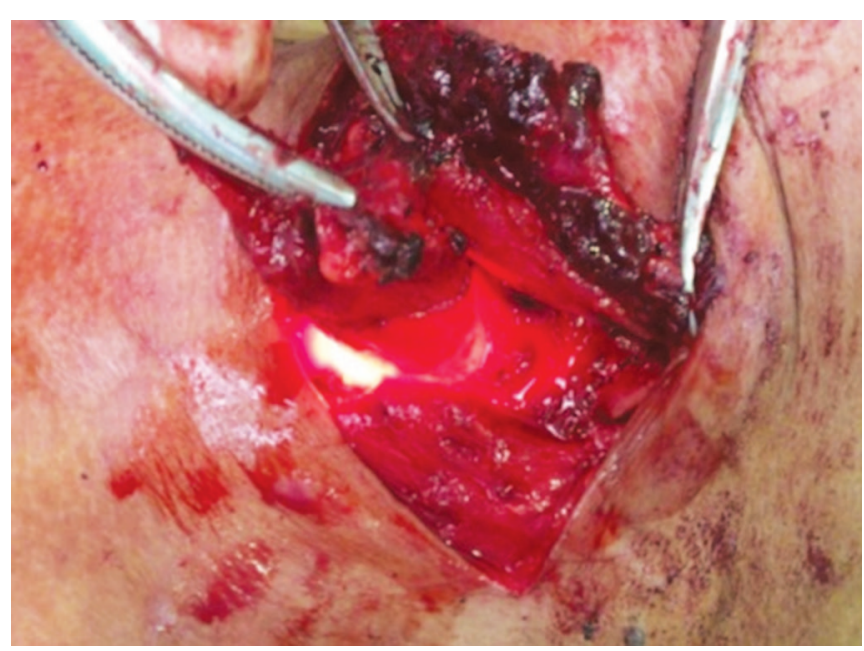

Fig. 3: The diverticulum to be arising anterolaterally and fixed to the base

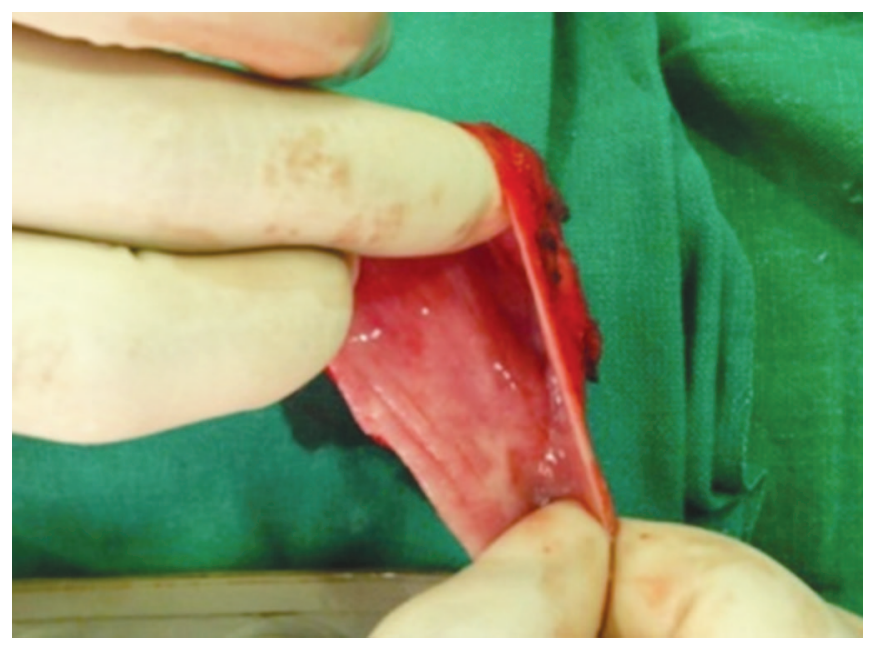

Fig. 4: The diverticulum after excision

described by Killian ${ }^{3}$ as corresponding to the area where the recurrent laryngeal nerve (RLN) enters the pharynx. This finding was later confirmed by Jamieson ${ }^{4}$ and is now termed
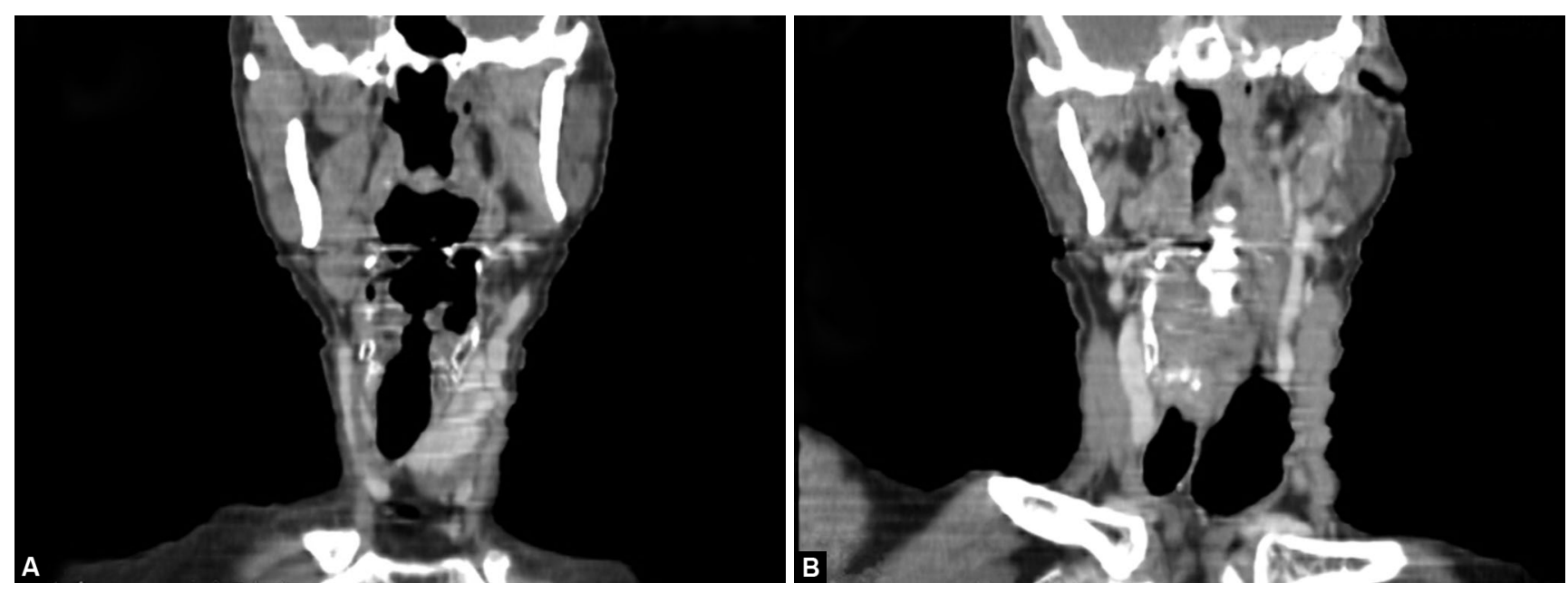

Figs 2A and B: Coronal sections of diverticulum below the level of cricopharyngeus muscle and inferiorly above the level of subclavian vein 
the Killian-Jamieson triangle. ${ }^{5}$ It is an uncommon cervical esophageal diverticulum located on the proximal lateral side of the cervical esophagus (also called as proximal lateral cervical diverticulum). KJD is rare in that the incidence of KJD is a fourth of that of ZD. Both ZD and KJD occur at sites of anatomic weakness in the hypopharynx or cervical esophagus near the cricopharyngeus muscle.

The pathogenesis of Killian-Jamieson diverticula is unknown. Tang et $\mathrm{al}^{6}$ hypothesized that Killian-Jamieson diverticula are the result of a functional outflow obstruction in the esophagus in much the same way that a Zenker's diverticula is believed to result from a functional outflow obstruction in the pharynx.

In a case series, ${ }^{1}$ patients with Zenker's diverticula were more likely to have symptoms (particularly suprasternal dysphagia) attributable to the underlying diverticulum than patients with Killian Jamieson diverticula, who usually were asymptomatic or had symptoms which were attributable to pharyngeal motility. Another feature that distinguished patients with Zenker's diverticulum from KJD was the greater risk of aspiration pneumonia, which occurred in $3(12 \%)$ of 26 cases. In all the 3 patients, ${ }^{1}$ there was reflux from the Zenker's diverticulum into hypopharynx with overflow aspiration on pharyngoesophagography. Other investigators ${ }^{7-10}$ have shown that patients with Zenker's diverticulum have a high prevalence of GERD on pharyngoesophagography.

A case series ${ }^{1}$ of 16 patients with Killian-Jamieson diverticula found 11 with symptoms. However, eight of these patients had abnormal pharyngeal motility or an abnormal oral phase of swallowing, which may have contributed or even caused these symptoms. The fact that Zenker's diverticula are, on average, larger than Killian-Jamieson diverticula, and are located in the posterior pharynx, may explain why these diverticula cause symptoms without the associated abnormal oral or pharyngeal motility that is seen with Killian-Jamieson diverticula.

Ekberg and Nylander ${ }^{2}$ originally described 17 patients with proximal lateral cervical esophageal diverticula. They classified the transient outpouchings as 'bulges' caused by a weakened esophageal wall and the persistent outpouchings as true diverticula with muscular elements. These transient bulges of the lateral wall of the proximal cervical esophagus described by Ekberg and Nylander are in fact pouches similar to those seen on pharyngoesophagography on the anterolateral walls of the hypopharynx below the level hyoid bone (known as lateral pharyngeal pouches). A case series of 16 patients ${ }^{1}$ which included only persistent outpouchings from the lateral wall of the proximal cervical esophagus similar to the four true KJD described by Ekberg and Nylander, found 20 such diverticula in 16 patients over 7 years. Seven were detected in six patients during 4 years period in which 26 cases of ZD were detected. Even this study indicates that ZD is nearly four times as common as KJD. The series also showed that $75 \%$ were left sided and 25\% were bilateral, whereas Ekberg and Nylander did not describe a greater frequency on either side of cervical esophagus.

Very few reports regarding the treatment of KillianJamieson diverticula have been cited in the literature. Rogers et al, ${ }^{11}$ approached the Killian-Jamieson diverticulum through a horizontal left neck incision. The diverticulum was then mobilized and transected with a surgical stapling device. No esophagomyotomy was performed. Tang et $\mathrm{al}^{6}$ performed a distal vertical diverticulotomy with a flexible endoscope. Using a needle-knife, the distal vertical diverticulotomy was performed approximately $10 \mathrm{~mm}$ vertically from the opening in the diverticulum. This resulted in a dissection of the circular esophageal muscle inferior to the diverticulum. Jacobson et al, ${ }^{12}$ and Kim et $\mathrm{al}^{13}$ also described an open transcervical excision for KJD to avoid inadvertent injury to RLN.

Lee et $\mathrm{al}^{14}$ presented a case of a symptomatic KJD that was successfully treated with the flexible endoscopic diverticulotomy using two new devices. An isolated-tip needle-knife papillotome (Iso-Tome) was used for the dissection of the tissue bridge of the diverticulum. And a flexible overtube with a modified distal end (a fitted overtube) was used for adequate visualization of the tissue bridge of the diverticulum and protection of the surrounding tissue during dissection of the tissue bridge. Their successful experience suggests that the flexible endoscopic diverticulotomy with the use of appropriate endoscopic devices can be a safe and effective method for the treatment of a symptomatic KJD.

Generally, ${ }^{1}$ endoscopic treatment is considered preferable in treating ZD smaller than $3 \mathrm{~cm}$. Endoscopic treatment has the possibility of occluded view when there is food or a foreign body in the diverticulum. The treatment of the KJD is more closely adjacent to the recurrent laryngeal nerves than ZD. For KJD, treatment other than endoscopic treatments are preferable. According to the previous studies, ${ }^{11,15}$ the safety of endoscopic treatment has not been established for KJD, due largely to the rarity of cases, and for ZD the recurrence rate of endoscopic treatment is 10 times higher than that of surgical treatment. Furthermore, myotomy should be adopted as a treatment for KJD to prevent recurrence.

At the base of the Killian-Jamieson's diverticula, the recurrent laryngeal nerves enter the pharynx. ${ }^{2}$ Because of the close proximity of the inferior laryngeal nerves to the base of these diverticula, we approached the diverticula transcutaneously instead of endoscopically to avoid injury 
to these nerves in the present case. We also performed an esophagomyotomy in addition to a diverticulectomy to relieve the potential functional obstruction in the circular esophageal muscle inferior to the diverticula. The circular esophageal muscle inferior to the diverticula may contribute to or cause its formation.

\section{CONCLUSION}

KJD are rare and poorly understood hypopharyngeal diverticula. These diverticula are unlikely to be distinguished from Zenker's diverticula based solely on their clinical presentations. Barium esophagography and a CT scan are helpful to make the diagnosis and differentiate between the two. Killian-Jamieson diverticula are the result of a functional esophageal obstruction. The symptoms observed in these patients may be due to an underlying abnormal oral and or pharyngeal phase of swallowing. Until its pathophysiology is better understood, we recommend that an esophagomyotomy be part of its surgical treatment. In addition, we recommend that these diverticula be approached transcutaneously to prevent a recurrent laryngeal nerve injury. According to the previous studies, the safety of endoscopic treatment has not been established for KJD, due largely to the rarity of case. Studies using esophageal myometry may help shed light onto the pathophysiology of these diverticula.

\section{REFERENCES}

1. Rubesin SE, Levine MS. Killian-Jamieson diverticula: Radiographic findings in 16 patients. AJR Am J Roentgenol 2001;177:85-89.

2. Ekberg O, Nylander G. Lateral diverticula from the pharyngoesophageal junction area. Radiology.1983;146:117-122.

3. Killian G. Über den Mund der Speiseröhre. Zeitschrift für Ohrenheilkunde 1908;55:1-44.

4. Jamieson EB. Illustrations of regional anatomy Edinburgh: E\&S Livingstone Ltd; 1934(Section 2):44.

5. Zaino C, Jacobson HG, Lepow H, Ozturk C. The pharyngoesophageal sphincter. Springfield: CC Thomas; 1950. pp. 29-144

6. Tang S, Tang L, Chen E, Myers LL. Flexible endoscopic KillianJamieson diverticulotomy and literature review. Gastrointest Endoscopy 2008;68:790-793.

7. Bachman AL, Seaman WB, Macken KL. Lateral pharyngeal diverticula. Radiology 1968;91:774-782.
8. Lindbichler, et al. Aspiration resulting from lateral hypopharyngeal pouches. AJR 1998;170:129-132.

9. Smiley, et al. Relationship between posterior pharyngeal pouch and hiatua hernia. Thorax 1970;25:725-731.

10. Delahunty, et al. The relationship of reflux esophagitis to pharyngeal pouch (Zenker's diverticulum). Laryngoscope 1971; 81:570-577.

11. Rogers PJ, Armstrong WB, Dana E. Killian-Jamieson diverticulum: a case report and a review of the literature. Ann Otol Rhinol Laryngology 2000;109:1087-1097.

12. Jacobson, et al. Killian Jamieson diverticulum: a case for open transcervical excision- Laryngoscope 2013 Feb;123(2):414-417. doi: 10.1002/lary.23639. Epub 2012 Nov 26.

13. Dong Chan Kim, et al. Surgical treatment of Killian Jameison diverticulum-Korean. J Thorac Cardiovasc Surg 2012 August;45(4):272-274. Published online 2012 August 3.

14. Lee, et al. Endoscopic diverticulotomy with an isolated-tip needle-knife papillotome (Iso-Tome) and a fitted overtube for the treatment of a Killian-Jamieson diverticulum. World J Gastroenterol 2008 November 14;14(42):6589-6592.

15. Eckberg O. Radiology of the Pharynx and the Esophagus. Berlin: Springer-Verlag; 2003.

\section{ABOUT THE AUTHORS}

\section{Prafulla Sakhare (Corresponding Author)}

Assistant Professor, Department of Otorhinolaryngology, Government Medical College and Hospital, Nagpur, Maharashtra, India Phone: 09373646387, e-mail: prafullasakhare@gmail.com

\section{Binhi Desai}

Postgraduate Resident, Department of Otorhinolaryngology Government Medical College and Hospital, Nagpur, Maharashtra India

\section{Bhagyashree Bokare}

Associate Professor, Department of Otorhinolaryngology, Government Medical College and Hospital, Nagpur, Maharashtra, India

\section{Seema Patel}

Associate Professor, Department of Otorhinolaryngology, Government Medical College and Hospital, Nagpur, Maharashtra, India

\section{Apurva Pawde}

Professor and Head, Department of Otorhinolaryngology, Government Medical College and Hospital, Nagpur, Maharashtra, India 